\title{
Detection of intracoronary thrombus by magnetic resonance imaging in patients with acute coronary syndrome
} Christian H Jansen*1, Divaka D Perera², Marcus R Makowski ${ }^{1}$, Andrea J Wiethoff ${ }^{1}$, Andreas Schuster ${ }^{1}$, Timothy Lockie ${ }^{1}$, Reza M Razavi ${ }^{1}$, Gerald F Greil ${ }^{1}$, Eike Nagel ${ }^{1}$, Simon Redwood ${ }^{2}$ and Rene M Botnar ${ }^{1}$

Address: ${ }^{1}$ King's College London, London, UK and ${ }^{2}$ Guy's \& St Thomas' Hosp/Kings Coll London, London, UK

* Corresponding author

from 13th Annual SCMR Scientific Sessions

Phoenix, AZ, USA. 21 -24 January 2010

Published: 21 January 2010

Journal of Cardiovascular Magnetic Resonance 20 I0, I2(Suppl I):O87 doi:I0.I I86/I532-429X-I2-SI-O87

This abstract is available from: http://jcmr-online.com/content/I2/SI/O87

(c) 2010 Jansen et al; licensee BioMed Central Ltd.

\section{Introduction}

Persistent intracoronary thrombus following plaque rupture is associated with an increased risk of subsequent myocardial infarction and mortality (Svilaas et al. N Engl J Med 2008;358:557-67). Clinically, coronary thrombus can only be visualized invasively by $x$-ray angiography (XRA), intravascular ultrasound, or angioscopy while preclinical studies with a fibrin-binding MR contrast agent have demonstrated the feasibility of non-invasive MR coronary thrombus imaging (Botnar et al. Circulation 2004). In addition, non-contrast enhanced T1 weighted MRI has been shown useful for direct imaging of carotid thrombus and intraplaque hemorrhage by taking advantage of the short T1 of methemoglobin present in acute thrombus and intraplaque hemorrhage (Moody et al. Circulation 2003;107:3047-3052).

\section{Purpose}

The aim of this study was to investigate the use of noncontrast enhanced magnetic resonance imaging for direct thrombus visualization (MRDTI) in patients with a recent acute coronary syndrome (ACS) (troponin $\mathrm{T}>1.0$ ).

\section{Methods}

19 patients (16 male, age $61.8 \pm 8.9$ ) underwent MRDTI within 24-72 h of presenting with ACS prior to invasive XRA. MRDTI (Fig. 1b+g) was performed using a navigatorgated free breathing and cardiac-triggered T1-weighted 3D inversion-recovery black-blood gradient-echo sequence without contrast administration.

\section{Results}

10 patients were found to have intracoronary thrombus on XRA +/- thrombus extraction $(4 \times \mathrm{LAD}, 2 \times \mathrm{LCX}, 4 \times$ RCA and 1PDA branch of RCA, one patient was diagnosed with thrombus formation in the proximal LAD and LCA) (Fig $1 \mathrm{e}+\mathrm{f}$ ) and 9 had no visible thrombus. TIMI Risk Score for ST elevation myocardial infarction (STEMI) showed a trend towards a higher in patients with thrombus detection (thrombus group: median 19.75 (1.6-35.9) vs. 2.2 $(0.8-35.9)$ for the non thrombus group, $\mathrm{p}=0.23)$. MRDTI (fused with magnetic resonance angiography, Fig. 1c+h) correctly identified thrombus in 10 of 11 patients (sensitivity: 91\%, PDA thrombus not detected) and correctly classified the control group of 9 patients without thrombus formation (specificity 100\%). Contrast-to-noise ratio (CNR) of thrombus as compared to the vessel lumen of non-affected segments was 9-fold increased $(64.1 \pm 12.4$ vs. $7.0 \pm 5.8, \mathrm{p}<0.01)$ and 2 -fold increased as compared to segments with thrombus $(26.2 \pm 7.2, \mathrm{p}<0.01)$. CNR of the non-affected segments ranged from 0.7-20.7 as compared to 45.7-87.6 for segments with thrombus.

\section{Conclusion}

MRDTI allows selective visualization of coronary thrombus in patients after myocardial infarction. A CNR cut-off value of 40 allows detection of intra-luminal thrombus 

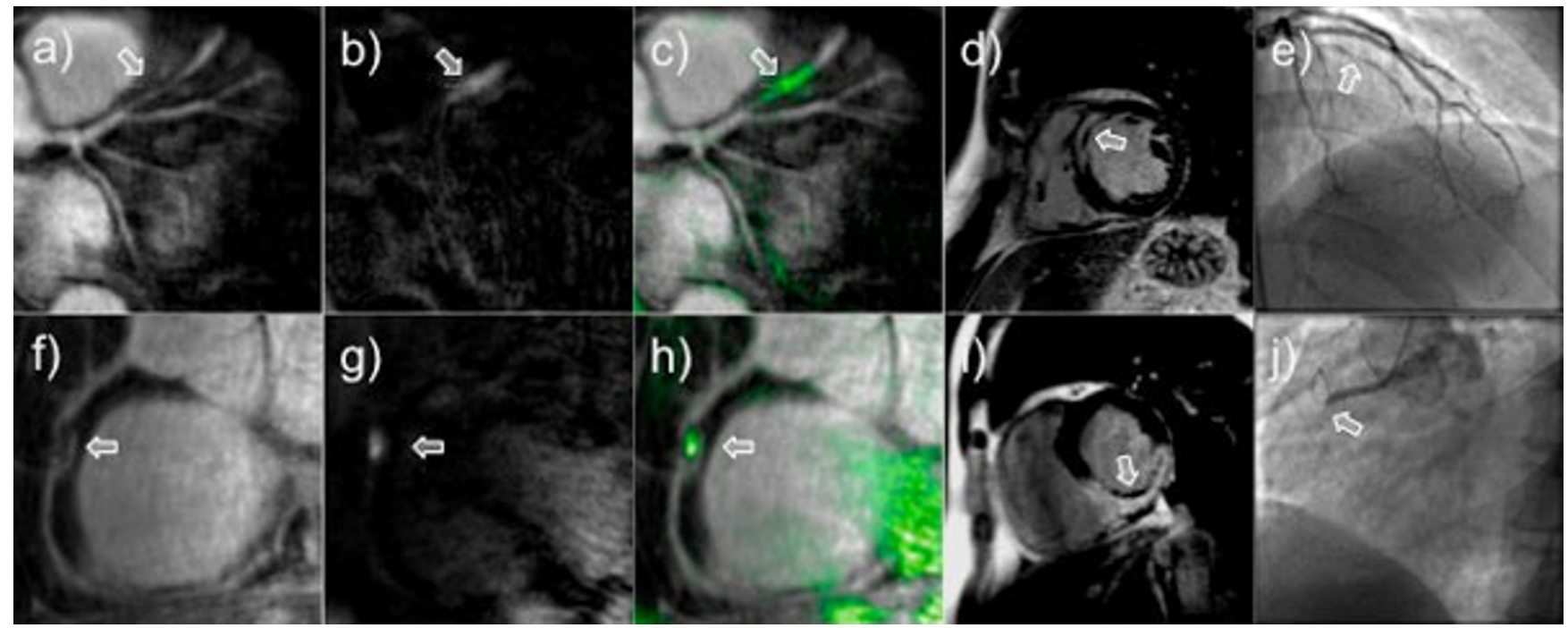

\section{Figure I}

a-e) 45 year old male with acutre anterior STEMI. Fusion (c) of MRA (a) and MRFTI (b) revealed mid LAD stenosis with thrombus formation (arrow). Late enhancement (LE) images showed non-transmural septal infarction with sub-endocardial microvascular obstruction (arrow). Corresponding XRA showed slwo flow with $90-95 \%$ mid LAD stenosis with thrombus (culpirt lesion). Thrombectomy performed with an Export catheter. $\mathrm{f}-\mathrm{j}$ ) 73 year old male with failed thrombolysis after acute inferior STEMI. Fusion (h) of MRA (g) and MRDTI demonstrate tight proxiomal to mid RCA stenosis with thrombus (arrow). LE images showed extensive transmural scarring of the infero-septal to infero-lateral segments with microvascular obstruction (arrow). XRA showed total occlusion of the RCA, collateralised by the left coronary arteries.

formation with a sensitivity and specificity of $100 \%$ in visible segments. This technique may be useful for direct coronary thrombus detection in patients with ACS or unstable angina.

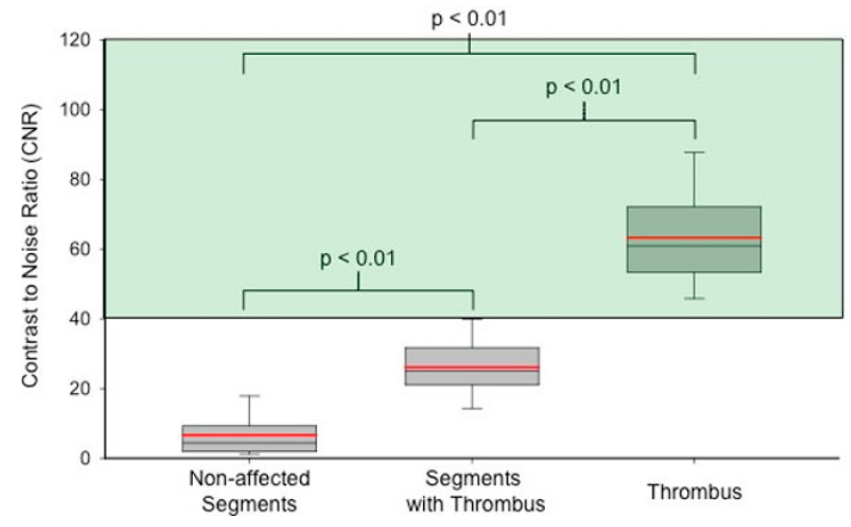

Figure 2

Box plot (red line indicating mean), 10 patients ( 10 male, age $62.4 \pm 9.9)$ with confirmated diagnosis of intra luminal thrombus $(n=10$, one patient with two thrombu in the proximal RCX and LAD). Contrast-tonoise ratio (CNR) of thrombus as compared to the vessel lumen of non-affected segments was 9 -fold increased $(64.1 \pm$ 12.4 vs. $7.0 \pm 5.8, p<0.01$ ) and 2 -fold increased as compared to segments with thrombus $(26.2 \pm 7.2, p<0.01)$.

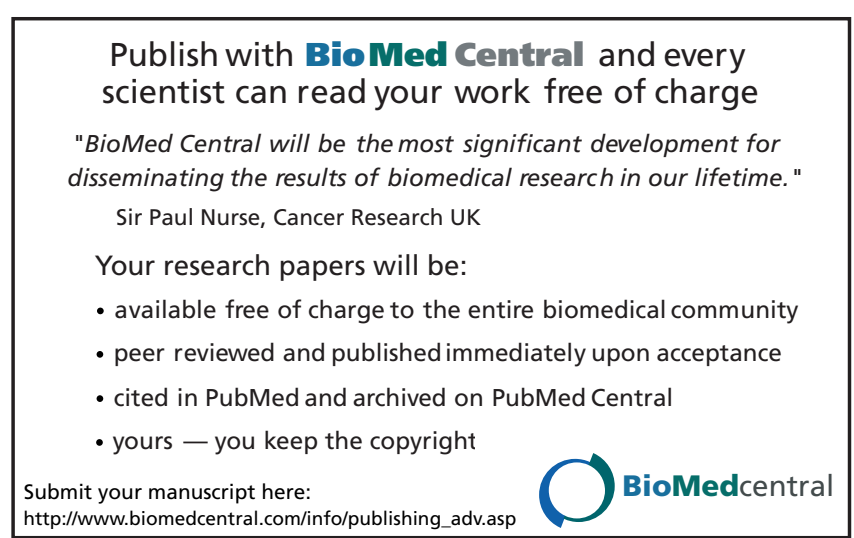

